\title{
Honey as a Natural Alternative for Formalin Fixative - A Systematic Review
}

\author{
Snega Thamilselvan ${ }^{1}$, Don K. R², Herald J. Sherlin³, Gifrina Jayaraj, Archana Santhanam5 \\ 1, 2, 3, 4, 5 Department of Oral Pathology, Saveetha Dental College and Hospitals, Saveetha Institute of Medical and \\ Techinical Sciences (SIMATS), Saveetha University, Chennai, Tamilnadu, India.
}

\section{ABSTRACT}

Fixation is the first step in histopathological tissue processing which is independently performed with $10 \%$ formaldehyde or formalin for over many decades. The fixation of tissues is performed to retain cellular components in their respective compartments and to withstand tissue processing, avoid decomposition, putrefaction and autolysis, an ideal fixative is required to impart mechanical rigidity. Due to the increasing concern about the potential carcinogenicity of formalin, opting for more secure choices is vital. One such safe natural alternative for fixation is honey. Honey has been proven to have medicinal properties that qualify it to be used as a fixative. The aim of this study is to do a systematic review on the efficacy of honey as a tissue fixative in histopathological laboratories. The articles for this review were searched from PubMed, Google search and manual search from the year 2009 - 2019 using the keywords Honey, natural substitute, natural alternative, neutral buffered formalin, $10 \%$ formalin, formaldehyde, tissue fixative and tissue fixation. The final of 9 articles were included in the review which compared the efficacy of honey as a natural alternative tissue fixative with the gold standard formalin. Once the articles to be reviewed were finalised, data was collected from each article, tabulated and was verified and interpreted. Honey as a fixative yielded satisfactory results with respect to cellular details and the results of maintaining the structural morphology of tissues were good. Yet, the staining properties of honey fixation did not yield an exact outcome. It, despite everything, stays to be in difficulty as differentiating results continue. Yet honey has likewise demonstrated comparable outcomes to that of formalin in histopathological tissue processing. There are also few noticeable limitations for using honey as fixative which is not observed in formalin. The formalin will continue to dominate as the best fixative in tissue processing when comparing all the parameters till newer alternatives are available for fixation. Still honey has the potential to be used as an alternative to formalin in histopathological laboratories. With an added benefit of honey being eco-friendly, easily available, cost effective, nontoxic and non-inflammable, it can also be used as an effective alternative.
Corresponding Author:

Dr. Don K.R.,

Reader, Department of Oral Pathology

\& Microbiology, Saveetha Dental

College and Hospitals, Saveetha

Institute of Medical and Technical

Sciences (SIMATS), Saveetha University,

162, Poonamelle High Road,

Velappanchavadi. Chennai-600077,

Tamilnadu, India.

E-mail:drkrdon@gmail.com

DOI: $10.14260 / j e m d s / 2021 / 82$

How to Cite This Article:

Thamilselvan S, Don KR, Sherlin HJ, et al. Honey as a natural alternative for formalin fixative - a systematic review. J Evolution Med Dent Sci 2021;10(06):369-374, DOI: 10.14260/jemds/2021/82

Submission 21-09-2020,

Peer Review 09-12-2020,

Acceptance 15-12-2020,

Published 08-02-2021.

Copyright (C) 2021 Snega Thamilselvan et al. This is an open access article distributed under Creative Commons Attribution License [Attribution 4.0 International (CC BY 4.0)]

\section{KEY WORDS}

Honey, Natural, Alternative, Tissue Fixative. 


\section{BACKGROUND}

Fixation in an imperative step before histopathological tissue processing for light microscopic examination in laboratories. The specimen is prepared first by 'Fixing' it using a chemical, formaldehyde and this prevents further deterioration and decay process (Autolysis) of the tissue specimen. ${ }^{1}$ Formaldehyde was discovered by a Russian Chemist Alexander M. Butlerov in 1859.1,2 Formaldehyde still remains as gold standard fixative in preservation of tissue specimens because of its ease of availability and its cost effectiveness. But the International Agency for Research on Cancer (IARC) classified formaldehyde as 'carcinogenic to humans' ${ }^{3}$ The U.S. Occupational Safety and Health Administration (OSHA, 2004) stated that the permissible exposure limit is $0.75 \mathrm{ppm}$ as an 8 hour time weighted average. ${ }^{4}$ Exposure of formalin more than this estimated value causes health ill effects such as irritation of eyes, nose, throat and allergic skin reaction. ${ }^{2}$ Also the primary criteria for long term specimen preservation with morphology \& anatomy preserved in its best possible condition is still a challenge. Hence, the need for a safe natural alternative leads to the innovative idea of usage "Honey" as fixative.

Honey is the natural sweet substance, produced by honeybees from the nectar of plants. Honey is a mixture of sugars and trace amounts of other compounds like chrysin, pinobanksin, vitamin C, catalase and pinocembrin. ${ }^{2}$ Honey has been proved to have dehydrating and preserving properties similar to gold standard formaldehyde which makes it ideal to be used as an eco-friendly fixative in pathological laboratory. ${ }^{2}$ There are also few properties exerted by honey other than fixation. It has a strong medicinal value by its antioxidant, antimicrobial, anti-inflammatory and antimutagenic effects. ${ }^{5}$ Many evidences suggest that honey turned out to be more effective in treating wounds. This was stated by Samarghandian et al. in his study; honey and health: a review of recent clinical research. ${ }^{5}$ With all these positive properties, many studies have attempted to explore the natural substance honey as a substitute for fixation of tissues with different concentrations.

The systematic reviews have an important role in modern health care. They are used to appraise evidence, information policy, construct guidelines and assess cost effectiveness of interventions. ${ }^{6}$ Our primary aim was to evaluate the efficacy of honey as a tissue fixative in histopathological laboratory and reporting the systematic review. This study can establish the original impact of eco-friendly, natural and safer alternatives for gold standard fixative, formaldehyde.

\section{METHODS}

\section{Search Strategy for Identification of Studies}

The search strategy was in accordance with the Cochrane guidelines for systematic reviews. The articles included in this study were extracted from PubMed and back references of the articles till the year 2019. The internet search was also done to obtain relevant articles of our interest. The studies which assessed and compared the efficacy of honey as fixative were included in this study. The titles of articles and abstracts were reviewed. The text of the selected articles was retrieved and further analysed.

\section{Search Methodology}

The search methodology applied in PubMed was using the following keywords: (((honey) and (C((natural alternative) OR natural substitute) or alternative) or substitute)) and ((c((formalin) or formaldehyde) or $10 \%$ formalin) or neutral buffered formalin) or formol)) and (((fixative) or tissue fixative) or tissue fixation). Filters: published in the last 10 years. In addition, internet search was also done using the keywords "honey" and "safer alternatives" and "fixative" and "formalin". Articles which had used honey as natural fixative with control groups were considered for the review.

\section{Inclusion Criteria}

- Original research articles done with natural bees honey as fixative as alternative to formaldehyde were included.

- Articles published in English language were included in the review.

- Articles published in the last 10 years (2009 - 2019) were included.

\section{Exclusion Criteria}

- Studies with no control group and review articles were excluded.

- Studies published in other languages were excluded.

- Studies that used natural fixatives other than honey were excluded.

- $\quad$ Studies conducted with different applications of honey other than tissue fixation were excluded from the review.

\section{Methods of Review}

The initial search yielded 158 results. Additional filters were added for restraining the search to last 10 years (2009 - 2019), yielding 128 results. 124 articles were excluded based on the exclusion criteria, title and abstract screening reviews. 4 articles were approved for full text review from PubMed search and an additional of 5 articles was included from Google search, manual search \& cross references. After the final full text review, 9 articles were included in this systematic review. Data was extracted from the full text articles and reviewed and extracted content. The Figure 1 presents the search flowchart.

\section{Data Extraction}

Once the articles to be reviewed were finalised, data was collected from each article, tabulated and was verified and interpreted.

\section{Outcomes}

The outcomes in this review examined and analysed the efficacy of the honey as a natural alternative for formalin fixative. 


\section{DISCUSSION}

The data was extracted and synthesized from $\mathrm{n}=9$ articles. Table 1 provides a summary of the included studies. The results of the $\mathrm{n}=9$ articles showed almost similar results. In the study conducted by Amirtaksha battacharya et al. ${ }^{7}$ analysed based on epithelial preservation, epithelial staining, connective tissue preservation, connective tissue staining for $24 \mathrm{hrs}, 48 \mathrm{hrs}, 72 \mathrm{hrs}$. With $100 \%$ honey \& $10 \%$ Neutral Buffered Formalin (NBF). It was observed that formalin gave better and comparable results than honey in fixation. Statistically significant differences were obtained between honey and formalin fixative in nuclear details and cytoplasmic staining ( $\mathrm{p}$ value $<0.01$ ).

The study conducted by Vidushi lalwani et al. ${ }^{2}$ analysed nuclear staining, cytoplasmic staining, tissue morphology, clarity of staining and uniformity of staining with $10 \%$ unprocessed honey, $10 \%$ processed honey \& $10 \%$ NBF. The nuclear staining of processed, unprocessed honey and NBF showed $100 \%$ staining efficiency. It was observed that $92 \%$ adequate staining in processed and unprocessed honey as compared to NBF in terms of cytoplasmic staining, $75 \%$ adequacy in tissue morphology in processed than unprocessed honey as compared to NBF, which showed $92 \%$. There was no statistical significant difference between tissues fixed in processed honey and unprocessed honey compared to formalin for adequacy of diagnosis. But the assessment of artefacts showed statistical significance between 2 groups of honey and formalin. (P value $=0.004$ )

Shankargouda patil et al.8,9 analysed the nuclear details, cytoplasmic details and staining qualities with $20 \%$ honey, 10 $\%$ NBF \& distilled water. The results of this study showed that cytoplasmic and nuclear details were satisfactory but showed areas of uneven staining of tissues preserved with honey. Honey was able to preserve the tissue over a period of $24 \mathrm{hrs}$.
But formalin fixation after $48 \mathrm{hrs}$. Significantly showed better results than honey. The same author has done a longitudinal study over 6 months and found that the cellular and nuclear clarity gradually decreased with evident shrinkage compared to formalin. No statistical significance between tissue fixed with honey and formalin was noticed ( $p$ value $=0.563$ ) at the end of 6 months.

The study conducted by Sri R et al.10,11 analysed based on H\&E (Haematoxylin and Eosin) staining and PAS (Periodic Acid-Schiff) \& Mason trichrome \& IHC (Immuno-HistoChemistry) with $10 \%$ honey \& $4 \%$ NBF. It was observed that good reasonable results were obtained in tissues fixed with honey with nuclear and cellular structures maintained. Also they conducted the study using high concentrations of buffered formalin $-10 \%$.

The tissues fixed in bee honey gave good comparable results with that of formalin fixed tissues in maintaining the nuclear and cellular structures. No statistically significant difference was seen, suggesting that honey was equivalent to NBF fixative in all parameters $(p>0.05)$.

M.I Udonkang et al. 12 analysed the nuclear \& cytoplasmic staining and preservation of tissue morphology with $20 \%, 50$ $\%, 70 \%, 90 \%, 100 \%$ of honey \& $10 \%$ buffered formalin for 48 hrs. The results showed $100 \%, 90 \%$, and $70 \%$ concentrations of honey gave good intensity and clear nuclear \& cytoplasmic staining with moderate preservation of tissue morphology. Statistical results showed tissues fixed in $20 \%$ and $50 \%$ honey showed putrefaction changes than $70 \%, 90$ $\%$ \& $100 \%$ honey after 72 hours and were statistically significant ( $p$ value 0.04). Minor differences in nuclear and cytoplasmic staining ( $p$ value $=0.391$ ), intensity and clarity of histopathological details ( $p$ value $=0.252$ ) among the honey fixed group were not statistically significant.

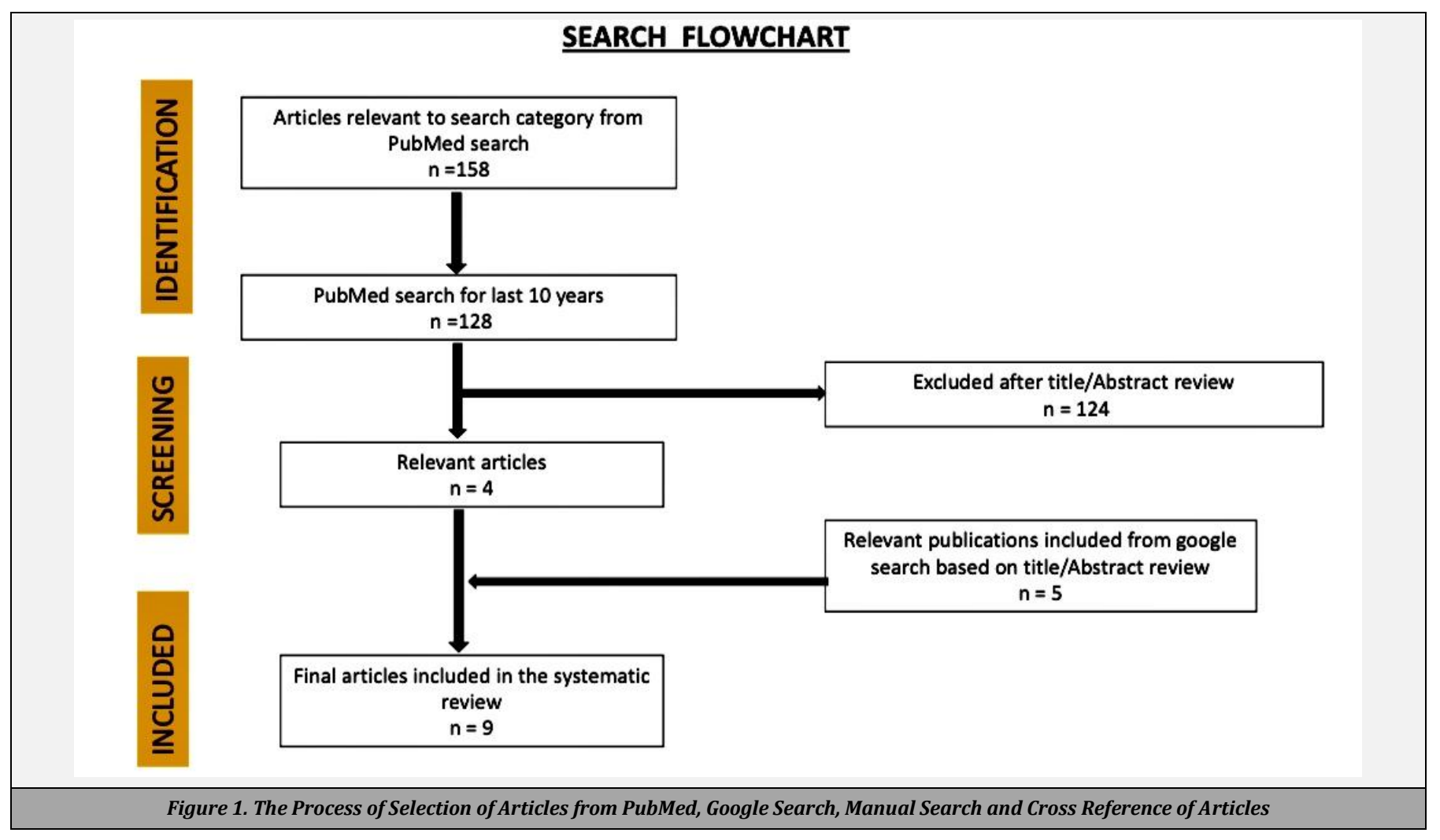




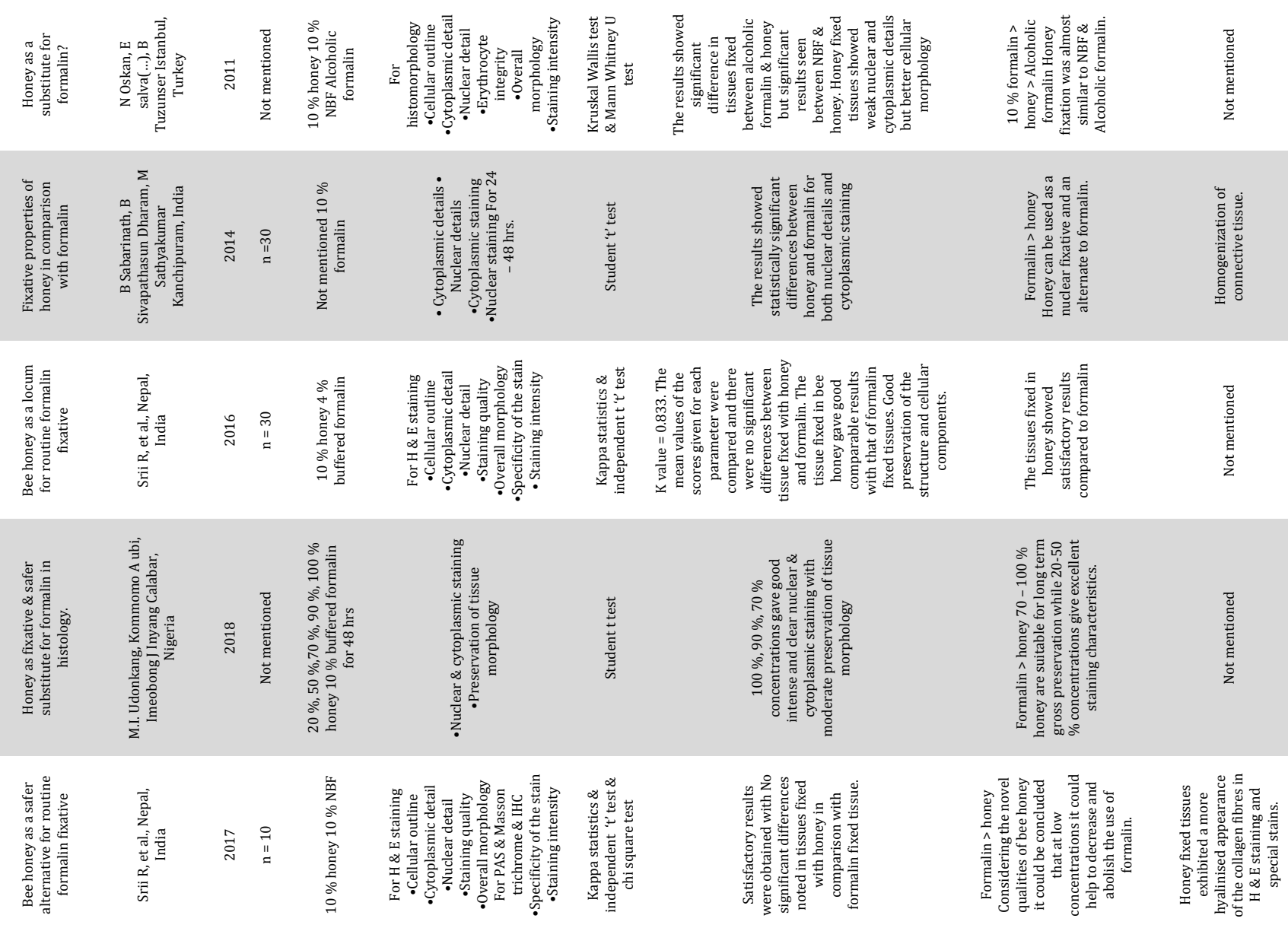

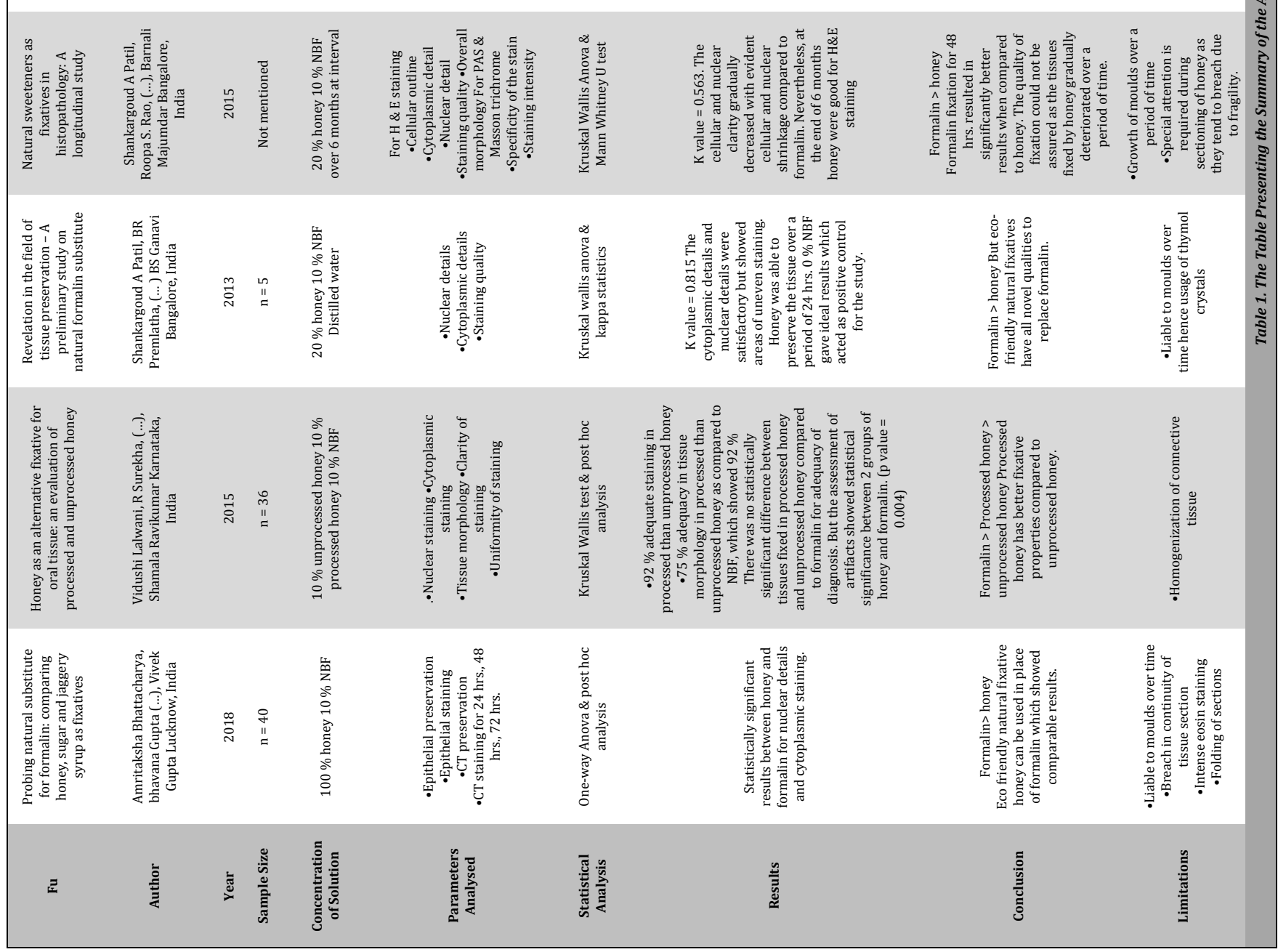


In the study conducted by Sabarinath et al. ${ }^{13}$ cytoplasmic $\&$ nuclear details \& staining for 24 - $48 \mathrm{hrs}$. were analysed with honey \& $10 \%$ NBF. It was observed that both honey and formalin showed statistically significant differences in nuclear details and cytoplasmic staining characteristics. The $p$ value for all the parameters analysed showed statistical significance $(\mathrm{p}<0.05)$.

In the study conducted by Oskan et al.14 analysed histomorphology characteristics based on cellular outline, cytoplasmic detail, nuclear detail, erythrocyte integrity, overall morphology \& staining intensity with $10 \%$ honey, 10 $\%$ NBF \& alcoholic formalin. The results of this study were observed to be honey fixed tissues showed weak nuclear and cytoplasmic details but better cellular morphology, but the preservation of morphology was similar to NBF. There were no significant differences ( $p>0.05$ ) among honey and formalin in terms of cytoplasmic details but in contrast significant differences were seen in tissue morphology $(p<0.05)$.

Honey can be used as a natural alternative for formalin because of its ease of availability. And also, honey has shown to possess many positive properties compared to other fixatives. The articles included in the study were reviewed for tissues fixed with different concentrations of honey compared with gold standard formalin based on various functional parameters. The microscopic characteristics are established by cytological and histological examination that provides diagnosis of certainty. ${ }^{15}$ Even though the main motives for choosing these substitutes is due to their ease of availability, eco-friendly nature, non-toxicity, cost-effectiveness and minimum armamentarium required, the preservation of tissue morphology is critical to provide an accurate diagnosis without any compromise in details. ${ }^{16}$

Studies showed that concentrations of honey used for fixation showed variations in results. Few studies done by Sri $\mathrm{R}$ et al, Vidushi Lalwani et al and Naziye Oskan et al.10,2, 14 showed that $10 \%$ honey provided comparable results to that of formalin with slightly minor histomorphological features but that does not interfere with diagnosis. Shankargouda Patil et al. identified $20 \%$ honey gave good staining efficacy and preservation of tissue..$^{9}$ The author Udonkang et al. also stated $20 \%-50 \%$ honey gave excellent tissue staining characteristics which was similar to that of NBF, also the authors mentioned its statistically significant.12 $70 \%-100 \%$ honey was found to be suitable for long term gross preservation. Thus, variations in concentrations of honey used as fixative provides much positive results in different aspects of preservation of tissues as compared to NBF.

The cellular details include nuclear and cytoplasmic details. There is a noteworthy contrast in results between safe alternative honey and NBF. The usage of honey as fixative has produced satisfactory results for the same when compared to NBF but none of the studies proved that honey was better compared to NBF in terms of nuclear \& cytoplasmic staining. Also, the cellular and nuclear clarity seem to be gradually decreased along with evident cellular shrinkage in tissues preserved in honey for an extensive period of time. But none of the studies had indicated any loss of cellular details after fixation of tissue with honey, which qualifies honey as a viable fixative and preservative for a shorter duration by the author Udonkang. ${ }^{12}$ In contrast, Shankargouda Patil et al. ${ }^{9}$ stated that long term preservation decreases the nuclear and cellular clarity when compared to formalin.
The structural morphology of the tissues is better preserved with honey fixation. The author Shankargouda Patil et al. ${ }^{9}$ noticed no evident shrinkage or swelling of the tissues over a period of 6 months when compared to NBF. There seems to be no imbibition of honey into the tissues. This could be because of its thick viscosity causing no swelling and since it's a non-chemical fluid there is no chemical reaction between the tissue and the fluid to cause any shrinkage. The mechanism of honey in the process of fixation is thought to be due to the conversion of carbohydrates to gluconic acid. The gluconic acid produced by the dehydrogenation reaction catalysed by gluconic oxidase. ${ }^{17}$ The other hypothesis which is thought to play a role in the process of fixation is due to the presence of fructose / glucose in honey which at low $\mathrm{pH}$ breaks down to form aldehydes. These aldehydes cross-link with amino acids present in the tissue (similar to the action of formaldehyde) resulting in the tissue fixation. ${ }^{18}$ So honey can be opted as a fixative for preservation of structural components of tissue similar to that of NBF but not suitable for longer period storage.

The staining of tissues can be used to highlight structural components as well as to enhance the tissue contrast and tissue differentiates for better visualization under light microscopy. ${ }^{19}$ The staining qualities include the nuclear and cytoplasmic staining intensity and clarity. The H\&E staining after tissue fixation was found to be intense \& clear in few studies and in contrast few other studies have shown uneven staining. Hence, the staining qualities for the tissues fixed with honey still remains to be a dilemma.

All studies have indicated consistently that formalin fixed tissues show preferred outcome over honey in every aspect. Yet honey has likewise demonstrated comparable outcomes to that of formalin in histopathological tissue processing. Thus, a natural substitute like honey which is economical, nontoxic and non-allergenic can be considered for an efficient use in laboratories.

Yet there are few limitations on using a natural honey as fixative. Liable to molds over time which causes breach in continuity of tissue section and makes the tissue fragile. There is also folding and homogenization of tissue sections. Honey fixed tissues exhibited a more hyalinised appearance of the collagen fibres in $\mathrm{H} \& \mathrm{E}$ staining and special stain.

\section{CONCLUSIONS}

Formaldehyde is routinely used in developing countries. Aspiration devices are rarely used. Safe disposal of toxic wastes may be non-existent or problematic. Therefore, finding and receiving another appropriate substitute such as honey with a performance which is almost similar to that of formalin helps in the elimination of formalin while fixing tissues for histopathological study.

Financial or other competing interests: None.

Disclosure forms provided by the authors are available with the full text of this article at jemds.com.

The authors would like to acknowledge the help and support rendered by the department of Oral Pathology \& Microbiology of Saveetha Dental College and Hospitals and the management for their constant assistance with the research. 


\section{REFERENCES}

[1] Rose CL, Hawks CA, Genoways HH, et al. Storage of natural history collections: a preventive conservation approach. Society for the Preservation of Natural History: American Institute for Conservation of Historic and Artistic Works, Smithsonian Institution, The George Washington University Museum Studies Program, 1995: p. 43-55.

[2] Lalwani V, Surekha R, Vanishree M, et al. Honey as an alternative fixative for oral tissue: an evaluation of processed and unprocessed honey. Journal of Oral and Maxillofacial Pathology 2015;19(3):342-7.

[3] Moelans CB, ter Hoeve N, van Ginkel JW, et al. Formaldehyde substitute fixatives: analysis of macroscopy, morphologic analysis and immunohistochemical analysis. American Journal of Clinical Pathology 2011;136(4):548-56.

[4] Conolly RB, Kimbell JS, Janszen D, et al. Human respiratory tract cancer risks of inhaled formaldehyde: dose-response predictions derived from biologicallymotivated computational modeling of a combined rodent and human dataset. Toxicological Sciences 2004;82(1):279-96.

[5] Samarghandian S, Borji A. Anticarcinogenic effect of saffron (Crocus sativus L.) and its ingredients. Pharmacognosy Research 2014;6(2):99-107.

[6] Cullis PS, Gudlaugsdottir K, Andrews J. A systematic review of the quality of conduct and reporting of systematic reviews and meta-analyses in paediatric surgery. PLoS One 2017;12(4):e0175213.

[7] Bhattacharyya A, Gupta B, Singh A, et al. Probing natural substitute for formalin: comparing honey, sugar and jaggery syrup as fixatives. Natl J Maxillofac Surg 2018;9(1):14-21.

[8] Patil S, Premalatha BR, Rao RS, et al. Revelation in the field of tissue preservation-a preliminary study on natural formalin substitutes. Journal of international oral health. J Int Oral Health 2013;5(1):31-8.
[9] Patil S, Rao RS, Ganavi BS, et al. Natural sweeteners as fixatives in histopathology: a longitudinal study. J Nat Sc Biol Med 2015;6(1):67-70.

[10] Srii R, Peter CD, Haragannavar VC, et al. Bee honey as a safer alternative for routine formalin fixation. Kathmandu Univ Med J 2017;15(60):308-12.

[11] Chettiar SR, Marla V. BEE honey as a locum for routine formalin fixative. International Journal of Scientific Research 2016;5(10):498-500. .https://www.researchgate.net/profile/Vinay_Marla/pu blication/308886725_BEE_Honey_As_A_Locum_for_Rout ine_Formalin_Fixative/links/582f03b608ae004f74be53c 1.pdf. Accessed 28 Jun 2020.

[12] Udonkang MI, Ubi KA, Inyang IJ. Honey as fixative and safer substitute for formalin in histology. Int J Med Lab Res 2018;3(3):11-7.

[13] Sabarinath B, Sivapathasundharam B, Sathyakumar M. Fixative properties of honey in comparison with formalin. J Histotechnol 2014;37(1):21-5.

[14] Özkan N, Şalva E, Cakalağaoğlu F, et al. Honey as a substitute for formalin? Biotech Histochem 2012;87(2):148-53.

[15] Baba AI, Câtoi C. Epithelial and melanocytic tumors of the skin. Chapter - 4. Comparative Oncology. Bucharest (RO): The Publishing House of The Romanian Academy 2007.

[16] Chittemsetti S, Nallamala S, Sravya T, et al. Natural substitutes for formalin: a boon to histopathology!! J Oral Maxillofac Pathol 2018;22(1):143.

[17] Ramachandran S, Nair S, Larroche C, et al. Gluconic Acid. Current Developments in Biotechnology and Bioengineering 2014: p. 577-99.

[18] Gunter M, Bryant P. Immunocytochemical evaluation of ductal carcinoma in breast after preservation in honey. J Histotechnol 2009;32(2):54-9.

[19] Alturkistani HA, Tashkandi FM, Mohammedsaleh ZM. Histological stains: a literature review and case study. Global Journal of Health Sci 2016;8(3):72-9. 